\title{
Calculations of labyrinth seals with and without diagnostic extraction in fluid-flow machines
}

\author{
Paweł Kaszowski, Ph.D., \\ Marek Dzida, Assoc. Prof., \\ Gdansk University of Technology, Poland \\ Piotr Krzyślak, Assoc. Prof., \\ Poznan University of Technology, Poland
}

\begin{abstract}
Labyrinth seals are essential components of steam turbine unit constructions. Two types of labyrinth seals can be named, the first of which is the seal without diagnostic steam extraction, and the second - with extraction. The distribution of flow parameters along the packing is affected remarkably by the average seal clearance. The presence of diagnostic extraction leads to the equation system which is determinable and can be inversed to calculate the average seal clearance Si. Analysing the obtained results leads to the conclusion that the information about this parameter provides opportunities to monitor the current state of the packing in real time. The applied calculation procedure bases on the de Saint-Venant equation. The article also includes a brief description of both types of seals.
\end{abstract}

Key words: labyrinth seals; Fanno curve; diagnostic extraction

\section{INTRODUCTION}

Steam turbines constitute fluid flow machines used for propelling ships and offshore oil platforms as well as for driving electric generators in power industry. Labyrinth seals are turbine elements responsible for minimization of heat steam leakage losses.

The labyrinth seals are classified as those with and without steam extraction. If a seal without extraction is selected then it is not possible to fully determine parameters occurring along the packing. However a seal with extraction gives such possibility. To this end a suitable calculation model is necessary.

\section{DESCRIPTION OF PACKING}

Among labyrinth seals two types are distinguished: the first is not fitted with a diagnostic extraction and the other is fitted with a diagnostic extraction.

Fig. 1 presents the schematic diagram of labyrinth seal without diagnostic extraction as well as distribution of pressure drop in particular seal chambers. Flux of mass flowing through the seal is determined by Fanno line presented in Fig. 2, and described by the function (1):

$$
\dot{m}=f\left(p_{0}, p_{k}, h_{0}, S_{n}, \mu\right)
$$

where:

$\dot{\mathrm{m}}$ - mass flux flowing through seal

$\mathrm{p}_{\mathrm{o}}-$ steam pressure before seal

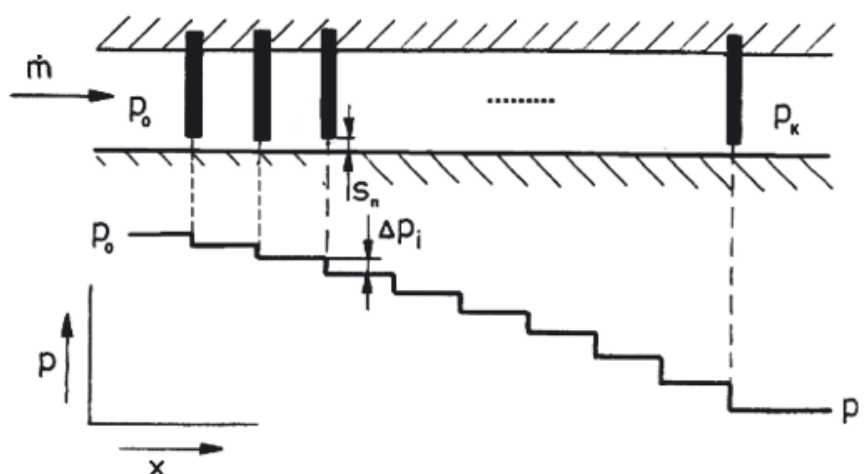

Fig. 1. Schematic diagram of labyrinth seal without diagnostic extraction

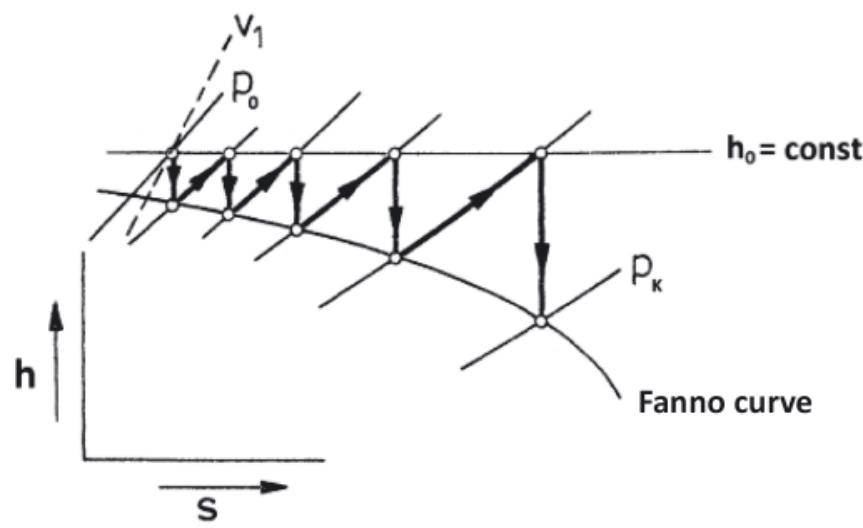

Fig. 2. Fanno line for seal without extraction 
$\mathrm{p}_{\mathrm{k}}-$ steam pressure behind seal

$\mathrm{h}_{\mathrm{o}}-$ initial medium enthalpy before seal

$\mathrm{s}_{\mathrm{n}}-$ mean clearance of seal

$\mu-$ flow rate for seal

The above given function which describes mass flux flowing through seal, is determined by mean clearance of seal. Values of mass flux and mean clearance are unknowns closely mutually connected. Despite the known values of pressure and temperature present before and behind the seal it is not possible to unambigously assess mass flux in the case if the mean value of clearance is not known. It means that to determine medium flow through such seal to know complete input data is necessary.

Lack of any initial parameter makes determining the value of interest not possible. If in the equation of mass flux through the seal one unknown appears then it is not possible to control state of wear of the seal.

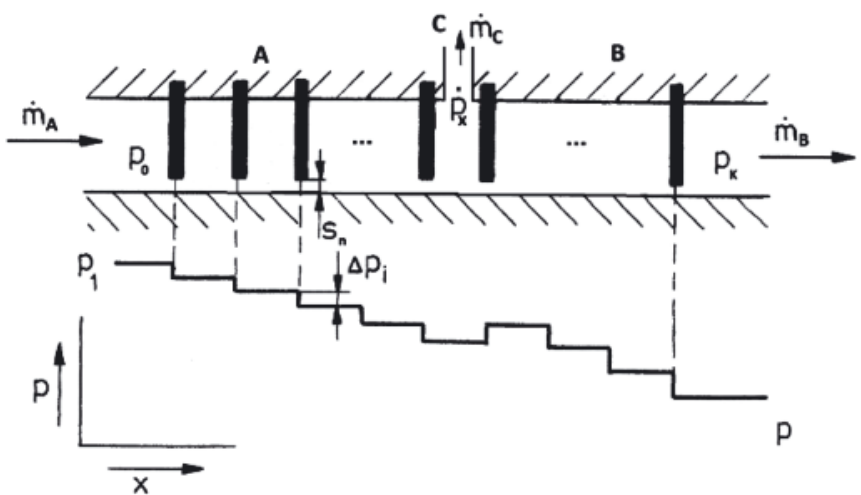

Fig. 3. Schematic diagram of labyrinth seal with diagnostic extraction

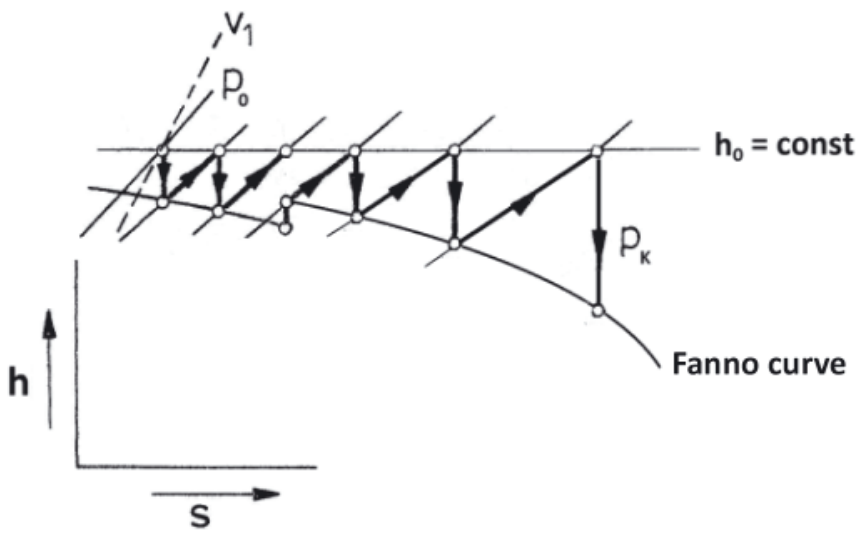

Fig. 4. Fanno line for seal with extraction

Fig. 3 presents the labyrinth seal with diagnostic extraction. An example run of Fanno curve for such packing is shown in Fig. 4.

The diagnostic channels are aimed at the disturbing of chocking process within the seal, realized by providing them an appropriate geometry, for instance by application of a nozzle. Location of steam extraction and pressure measurement channels is to be so optimized as to ensure, on the basis of thermodynamic and fluid mechanic relationships, maximum possible accuracy and sensitivity of diagnostic apparature. Role of extraction channels is more thoroughly described in the publication [3].

A seal can be divided into segments. The segment $\mathrm{A}$ is a seal fragment between the inlet to packing and diagnostic extraction channel. The next segment, marked B, is situated between the diagnostic channel and the outlet from the seal. And, the segment $\mathrm{C}$ comprises the mass extraction channel. To the labyrinth packing with diagnostic extraction the following set of equations is applicable:

$$
\begin{gathered}
\dot{m}_{C}=\dot{m}_{A}-\dot{m}_{B} \\
\dot{m}_{A}=f\left(p_{0}, p_{x}, h_{0}, S_{n}, \mu\right) \\
\dot{m}_{B}=f\left(p_{x}, p_{k}, h_{0}, S_{n}, \mu\right)
\end{gathered}
$$

where:

$\dot{\mathrm{m}}_{\mathrm{A}}-$ mass flux flowing through the seal segment A

$\dot{m}_{B}-$ mass flux flowing through the seal segment B

$\dot{\mathrm{m}}_{\mathrm{C}}-$ mass flux flowing through the diagnostic extraction channel

$\mathrm{p}_{\mathrm{o}} \quad-$ steam pressure before seal

$p_{k}-$ steam pressure behind seal

$\mathrm{p}_{\mathrm{x}} \quad-$ steam pressure occurring in the space from which $\dot{\mathrm{m}}_{\mathrm{C}}$ mass flux is discharged

$\mathrm{h}_{\mathrm{o}} \quad-$ initial medium enthalpy before seal

$\mathrm{s}_{\mathrm{n}}-$ mean clearance of seal

$\mu \quad-$ flow rate for seal

If a value of $\dot{\mathrm{m}}_{\mathrm{C}}$ steam mass flux through extraction channel is known then the above given set of equations is solvable and it makes it possible to determine value of mean seal clearance and values of leakges through the segments A and B. Owing to the knowlege of parameters occurring in a seal its wear state can be determined and consequently its overhaul schedule planned. In the case of a seal without diagnostic channel such task is not feasible.

\section{CALCULATIONS OF STEAM THERMODYNAMIC PARAMETERS ALONG THE LABYRINTH SEAL WITHOUT DIAGNOSTIC EXTRACTION}

The gas thermodynamic parameters were determined in particular points of the seal in accordance with Fig. 1.

The pressure drop in successive gaps of the seal, assumed linear, is calculated by means of the following formula:

$$
\Delta p=\frac{p_{0}-p_{k}}{z}
$$

where:

$\mathrm{z} \quad-\quad$ number of seal stages

The seal gap cross-section area $\mathrm{A}$ is determined by the mean seal clearance $S_{n}$ and seal diameter $D$ :

$$
A=\pi D S_{n}
$$

Value of the specific steam volume and the steam enthropy $\mathrm{s}_{\mathrm{o}}$ was read from steam tables. This way all the parameters necessary for determining location of the point 0 at the $h-s$ diagram (see Fig. 5), were found.

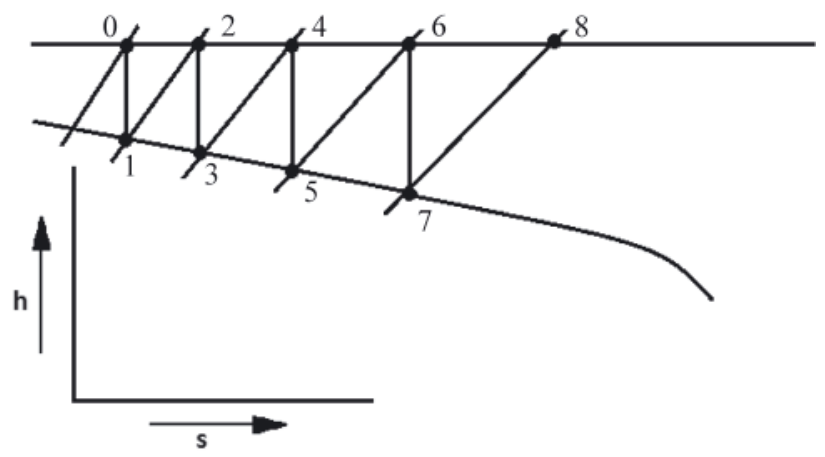

Fig. 5. Way of determining Fanno line for a seal 
Change of steam parameters during isenthropic process of expansion between the points $(0-1)$ was determined by using the flow equation as follows:

$$
\frac{c}{v}=\frac{\dot{m}_{n}}{A}=K=\text { idem }
$$

where:

$v$ - specific volume of steam flowing through a seal segment

Gas velocity at outlet from the seal segment is determined by the equation:

$$
c=v \frac{\dot{m}_{n}}{A}
$$

Drop of enthalpy in the seal segment by the equation:

$$
\Delta h=\frac{c^{2}}{2}
$$

or, if the seal parameters are taken into account, by the equation:

$$
\Delta h=v^{2} \frac{\dot{m}_{n}^{2}}{2 A^{2}}
$$

By taking into account the enthalpy drop in the first seal segment the location of the point 1 at the $h-s$ diagrams, i.e. the outlet from the first segment, is obtained.

The process between the points $1-2$ was assumed the isobaric braking of the flow from the contraction $\left(\mathrm{p}_{1}=\mathrm{p}_{2}\right)$. This way kinetic energy conversion into heat by lowering flow velocity due to friction with simultaneous lack of environmental heat exchange, was asssumed. During the process the steam enthalpy, at outlet from the seal, increases up to the value equal to that before the medium inlet to the seal.

$$
h_{2}=h_{1}+\frac{c_{2}^{2}}{2}=h_{0}
$$

Before each successive contraction the medium is assumed to be of the constant enthalpy value equal to initial one. Steam temperature value is read from steam tables. Location of all the points along the seal was determined by making use of the assumption that the mass braking occurs in the isobaric process, and of the prior calculated pressure distribution in the seal, as well as the presented way of calculating.

Next, values of mass flow rate in every gap of the seal were determined by means of de'Saint - Venant equation:

$\dot{m}_{i}=A \sqrt{2 g \frac{\kappa}{\kappa-1}\left[\left(\frac{p_{i+1}}{p_{i}}\right)^{\frac{2}{\kappa}}-\left(\frac{p_{i+1}}{p_{i}}\right)^{\frac{\kappa-1}{\kappa}}\right] \frac{p_{i}}{v_{i}}}$

where:

i $\quad-\quad$ number of seal segment

The steam flow differences in particular seal stages were this way obtained. As results from the calulations the pressure distribution in successive seal segments is not unambigous. To the seal calculations some pressure corrections should be introduced.

\section{PRESSURE CORRECTION}

Mass flux flowing through every contraction is described by Eq.(10) from which it results that flow through every gap is directly dependent on the pressure before the obstruction, $p_{i}$, and that behind it, $\mathrm{p}_{\mathrm{i}+1}$. For the assumed linear pressure drop the mass flux flowing through every contraction is not constant. For the searched-for pressure distribution the mass flux flowing through every contraction should be the same, which results from the mass conservation law. In order to obtain it a pressure correction should be made for every chamber.

By making use of the de Saint - Venant equation for $\mathrm{z}$ stages of the packing the set of $(z-1)$ equations describing the leakage from the whole seal, is obtained.

The relation (10) is developed into Taylor series, but only up to $1^{\text {st }}$ order derivatives, as the so obtained accuracy is sufficient; this way the linear equation of the following form is found:

$$
d \dot{m}=\frac{\partial \dot{m}}{\partial p_{i}} d p_{i}+\frac{\partial \dot{m}}{\partial p_{i+1}} d p_{i+1}
$$

This operation makes it possible to determine the pressure corrections $\Delta \mathrm{p}_{1}, \Delta \mathrm{p}_{2}, \ldots, \Delta \mathrm{p}_{\mathrm{z}}$. The partial derivatives appearing in Eq. (11) are as follows:

$$
\begin{aligned}
& \frac{\partial \dot{m}}{\partial p_{i}}=\frac{A}{2} \sqrt{2 g \frac{\kappa}{\kappa-1} \frac{1}{v_{i} p_{i}}} \frac{1}{\sqrt{\left(\frac{p_{i+1}}{p_{i}}\right)^{\frac{2}{\kappa}}-\left(\frac{p_{i+1}}{p_{i}}\right)^{\frac{\kappa+1}{\kappa}}}} \times \\
& \quad \times\left[\left(1-\frac{2}{\kappa}\right)\left(\frac{p_{i+1}}{p_{i}}\right)^{\frac{2}{\kappa}}+\left(\frac{\kappa+1}{\kappa}-1\right)\left(\frac{p_{i+1}}{p_{i}}\right)^{\frac{\kappa+1}{\kappa}}\right]
\end{aligned}
$$

$$
\begin{aligned}
\frac{\partial \dot{m}}{\partial p_{i+1}} & =\frac{A}{2} \sqrt{2 g \frac{\kappa}{\kappa-1} \frac{p_{i}}{v_{i}}} \frac{1}{\sqrt{\left(\frac{p_{i+1}}{p_{i}}\right)^{\frac{2}{\kappa}}-\left(\frac{p_{i+1}}{p_{i}}\right)^{\frac{\kappa+1}{\kappa}}}} \times \\
& \times\left(\frac{1}{p_{i}}\right)\left[\frac{2}{\kappa}\left(\frac{p_{i+1}}{p_{i}}\right)^{\frac{2}{\kappa}}+\frac{\kappa+1}{\kappa}\left(\frac{p_{i+1}}{p_{i}}\right)^{\frac{\kappa+1}{\kappa}}\right]
\end{aligned}
$$

The general form of Eq. (11), on the assumption that the increments $\Delta \mathrm{p}$ and $\Delta \dot{\mathrm{m}}$ are sufficenty small, describes a change of mass flux flowing through $i$-th contraction depending on pressure changes before and behind the contraction:

$$
\Delta \dot{m}_{i}=\frac{\partial \dot{m}}{\partial p_{i}} \Delta p_{i}+\frac{\partial \dot{m}}{\partial p_{i+1}} \Delta p_{i+1}
$$

Eq. (13) is formed separately for every gap in the seal:

$$
\begin{gathered}
\Delta \dot{m}_{1}=\frac{\partial \dot{m}_{1}}{\partial p_{0}} \Delta p_{0}+\frac{\partial \dot{m}_{1}}{\partial p_{1}} \Delta p_{1} \\
\ldots \dot{m}_{z}=\frac{\partial \dot{m}_{n}}{\partial p_{i-1}} \Delta p_{i-1}+\frac{\partial \dot{m}_{n}}{\partial p_{i}} \Delta p_{i}
\end{gathered}
$$

and also:

$$
\begin{gathered}
\Delta \dot{m}_{1}=\dot{m}_{1}-\dot{m}_{2} \\
\Delta \dot{m}_{i}=\dot{m}_{i}-\dot{m}_{i+1}
\end{gathered}
$$

In order to obtain a correct pressure distribution in the packing the same mass flux flowing through every contraction 
should be achieved. After completing the calculations the difference $\Delta \dot{\mathrm{m}}_{\mathrm{i}}=\dot{\mathrm{m}}_{\mathrm{i}}-\dot{\mathrm{m}}_{\mathrm{i}+1}$ is to be close to zero. This way the set of $(z-1)$ linear equations is obtained:

$$
\begin{gathered}
\dot{m}_{1}-\dot{m}_{2}=\frac{\partial \dot{m}_{1}}{\partial p_{0}} \Delta p_{0}+\frac{\partial \dot{m}_{1}}{\partial p_{1}} \Delta p_{1} \\
\dot{m}_{i}-\dot{m}_{i+1}=\frac{\partial \dot{m}_{i}}{\partial p_{i-1}} \Delta p_{i-1}+\frac{\partial \dot{m}_{i}}{\partial p_{i}} \Delta p_{i}
\end{gathered}
$$

The above given set of equations is solved by transforming the task to the matrix problem as follows:

$$
\boldsymbol{A} \cdot \boldsymbol{X}=\boldsymbol{B}
$$

where the square matrix $\mathbf{A}$ is built of the components of Eq. (17), except of the unknowns $\Delta \mathrm{p}_{1}$, arranged as follows:

$$
\boldsymbol{A}=\left[\begin{array}{ccc}
\frac{\partial \dot{m}_{1}}{\partial p_{1}} & \cdots & 0 \\
\vdots & \ddots & \vdots \\
0 & \cdots & \frac{\partial \dot{m}_{z-1}}{\partial p_{z-1}}
\end{array}\right]
$$

The vector $\mathbf{X}$ is built of the searched-for values of the unknown pressure drops $\Delta \mathrm{p}_{1}, \Delta \mathrm{p}_{2}, \ldots, \Delta \mathrm{p}_{\mathrm{z}}$ :

$$
\boldsymbol{X}=\left[\begin{array}{c}
\Delta p_{1} \\
\vdots \\
\Delta p_{z-1}
\end{array}\right]
$$

And, the matrix $\mathbf{B}$ is composed of the mass flux differences:

$$
\boldsymbol{B}=\left[\begin{array}{c}
-\left(\dot{m}_{1}-\dot{m}_{2}\right) \\
\vdots \\
-\left(\dot{m}_{z-1}-\dot{m}_{z}\right)
\end{array}\right]
$$

Next, in order to determine the unknowns $\Delta \mathrm{p}_{1}, \Delta \mathrm{p}_{2}, \ldots$, $\Delta \mathrm{p}_{\mathrm{z}-1}$ from the set of matrix equations the inverse matrix is applied:

$$
\boldsymbol{X}=\boldsymbol{A}^{-1} \cdot \boldsymbol{B}
$$

The so calculated values constitute corrections for the pressure drop initially assumed linear, in successive labyrinth packing chambers. The calculations are performed by using iterations up to reaching an assumed accuracy of calculations.

In the above presented way were performed calculations for the interbody seal of the HP turbine of 200MW power and the following parameters:

Pressure before the seal

$\begin{array}{lll}\mathrm{p}_{\mathrm{o}} & 9.861 & \mathrm{MPa} \\ \mathrm{t}_{\mathrm{o}} & 522.5 & { }^{\circ} \mathrm{C} \\ \mathrm{h}_{\mathrm{o}} & 3433 & \mathrm{~kJ} / \mathrm{kg} \\ \mathrm{p}_{2} & 4.204 & \mathrm{MPa} \\ \dot{\mathrm{m} n} & 2.228 & \mathrm{~kg} / \mathrm{s} \\ \mathrm{D} & 475 & \mathrm{~mm} \\ \mathrm{~S}_{\mathrm{n}} & 1.0 & \mathrm{~mm} \\ \mathrm{z} & 80 & \end{array}$

Steam temperature before the seal

Enthalpy before the seal

Pressure behind the seal

Steam flow rate through the seal

Shaft diameter

Nominal clearance of the seal

Number of seal stages

Fig. 6 and 7 present diagrams of pressure distribution and mass flux flowing through the seal. The results before introduction of the correction are marked red, and those after the correction - blue. It can be observed that the pressure distribution, as results from Eq. (10), is directly connected with the pressure occurring in the chambers before and behind seal stage. The corrections were performed iteratively up to reaching the accuracy of $\Delta \mathrm{m} / \mathrm{m}_{\mathrm{n}}=10^{-3}$.

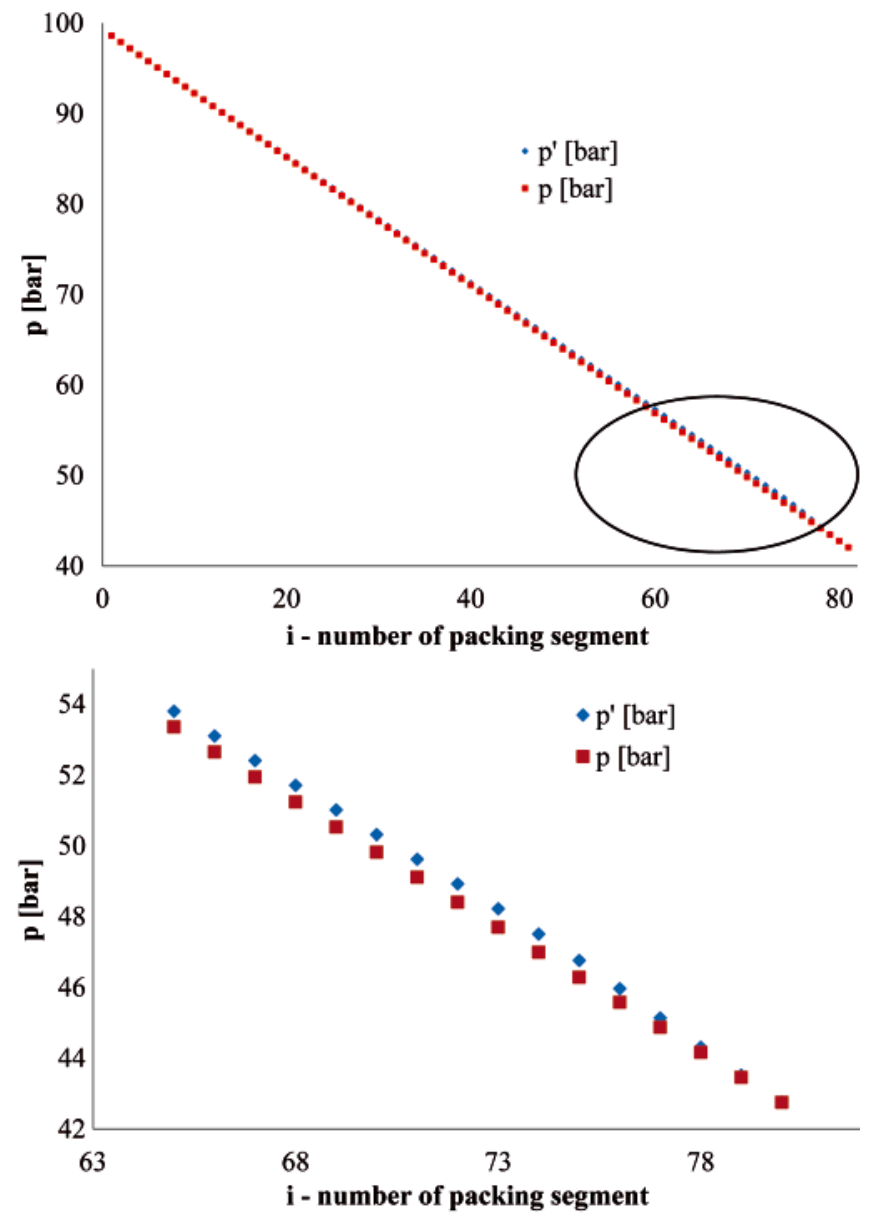

Fig. 6. Comparison of pressure distributions along the seal before and after introduction of corrections; $\boldsymbol{p}$ - pressure distribution before correction, p'-pressure distribution after introduction of correction

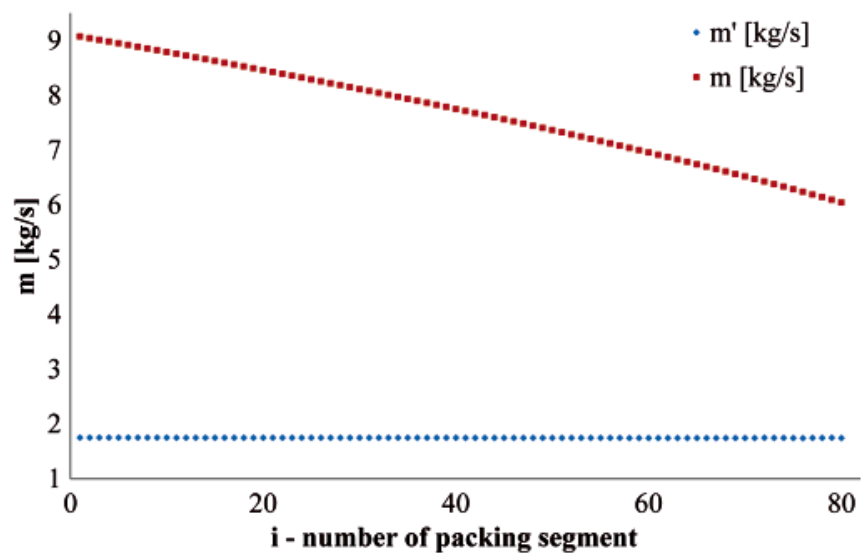

Fig. 7. Comparison of mass fluxes flowing through the seal gaps; $\boldsymbol{m}$ - before correction, $\boldsymbol{m}$ '- after introduction of correction

Calculations of steam thermodynamic parameters along the packing with diagnostic extraction were performed. The additional steam extraction of $0.4 \mathrm{~kg} / \mathrm{s}$ flow rate will cause disturbances of medium flow through seals by extracting a part of steam from space between contractions, located between $70^{\text {th }}$ and $71^{\text {st }}$ seal stage. It is assumed that the temperature and pressure occurring in the space from which the mass flux is extracted, is not changeable, i.e. the isobaric decreasing of gas velocity occurs. The smaller steam flux flowing through 
successive gaps will cause a smaller pressure drop in the gap at isenthropic expansion. It will result in a noticeable increase of pressure in the place where the diagnostic extraction is located (Fig. 8).
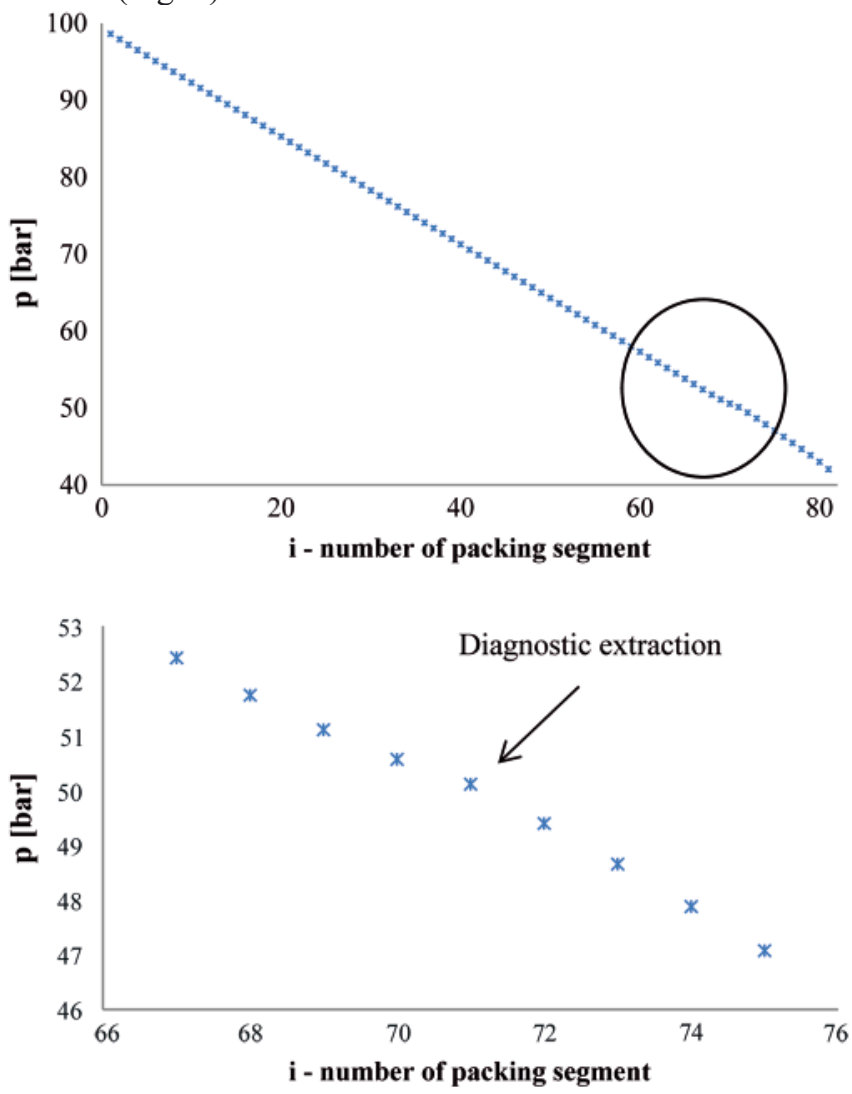

Fig. 8. Pressure distribution in the seal with diagnostic extraction

At the remaining segment of the packing, i.e. that measured from the extraction up to the end of the seal, flow drag of the decreased mass flux will be lower because of lower flow velocities. The mass flux flowing through the seal without diagnostic extraction as well as that for the seal with extraction is presented in Fig. 9. In the case of the seal with diagnostic extraction, behind $70^{\text {th }}$ seal stage can be observed a drop in value of the mass flux flowing through the seal by the value extracted through the diagnostic channel. The spread of results for this seal fragment is associated with the assumed calculation accuracy and computing power of the used computer. It does not result from accuracy of the calculation method.

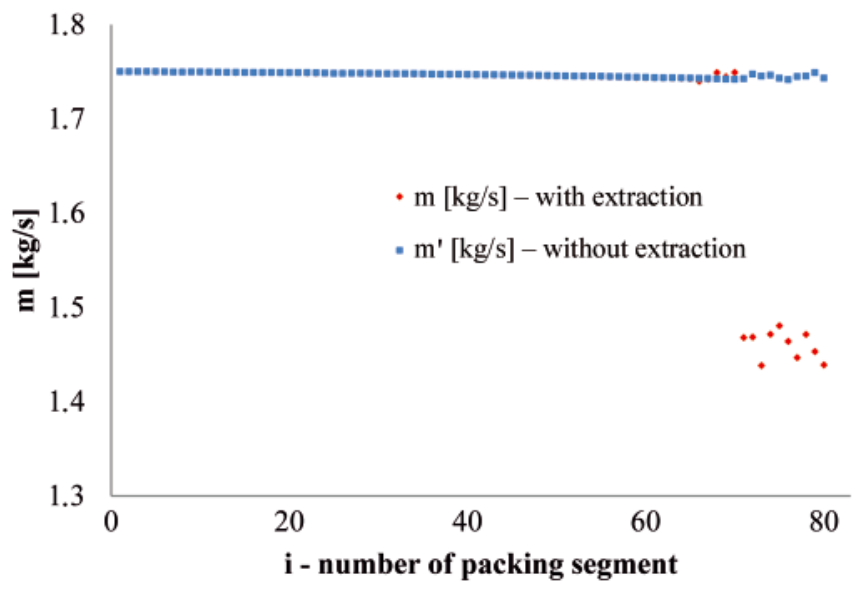

Fig. 9. Mass flux flowing through the seal with and without diagnostic extraction

The run of Fanno line was presented for both the cases in Fig. 10. The Fanno line for the seal without diagnostic extraction is marked black, in red is depicted the Fanno line for the packing with diagnostic extraction, and in blue colour is shown the inital enthalpy value.

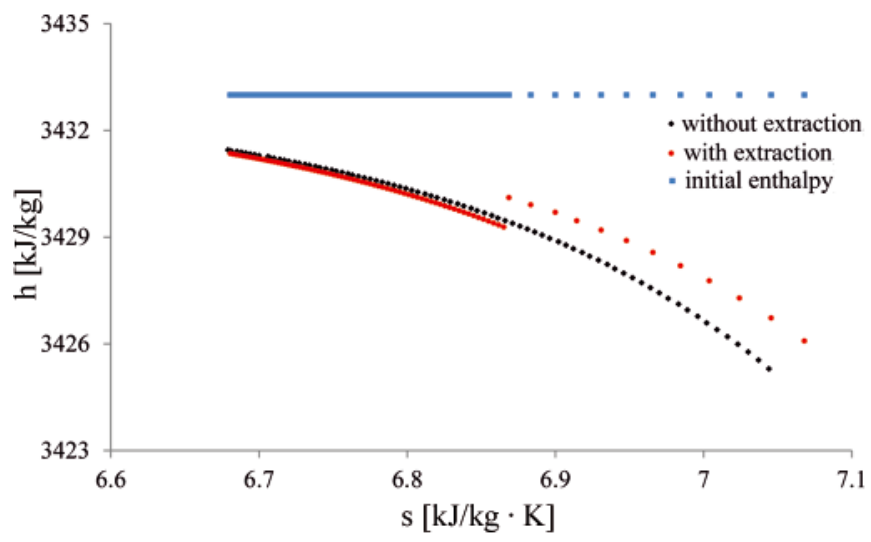

Fig. 10. Fanno line for labyrinth seal without and with diagnostic extraction

\section{SUMMARY}

In the case of the labyrinth seal with extraction the elaborated set of equations is determinate. It makes it possible to exactly determine values of parameters occurring along the seal, in contrast to that without extraction. This is the unambigous equation system which can be inverted in order to determine the mean seal clearance: $\mathrm{Si}=\mathrm{f}\left(\mathrm{p}_{\mathrm{o}}, \mathrm{p}_{\mathrm{x}}, \mathrm{h}_{\mathrm{o}}, \dot{\mathrm{m}}, \mu\right)$.

Knowledge of this parameter makes real-time diagnosing state of labyrinth seal, possible. It allows for planning repair and maintenance time without necessity of stopping the turbines, as well as for determining state of seal wear.

\section{BIBLIOGRAPHY}

1. Chmielniak T.: Steam turbines: theoretical background (in Polish). Wydawnictwo Politechniki Śląskiej (Publishing House of Silesian University of Technology),Gliwice, 1998

2. Kaszowski P.: Analysis of operation of labyrinth seals with extraction (in Polish). M.Sc. Thesis, Gdańsk University of Technology, Department of Energy Engineering and Industrial Apparatures, Gdańsk, 2011

3. Krzyślak P., Winowiecki M.: A method of diagnosing labyrinth seals in fluid-flow machines. Polish Maritime Research 3(57) Vol. 15, 2008, pp. 38-41

4. Perycz S.: Steam and gas turbines (in Polish). Wydawnictwo Politechniki Gdańskiej (Publishing House of Gdańsk University of Technology), Gdańsk 1988

5. Trütnovsky K.: Berührungsfreie Dichtungen, Grundlagen und Anwendungen der Strömung durch Spalte und Labirynthe (in German). VDI - VERLAG bh; (Verlag des Vereins Deutscher Ingeniuere), Düsseldorf, 1964

\section{CONTACT WITH THE AUTHORS}

Paweł Kaszowski, Ph.D.

Marek Dzida, Assoc. Prof.

Faculty of Ocean Engineering and Ship Technology

Gdansk University of Technology

Narutowicza 11/12

80-233 Gdansk, POLAND

e-mail: dzida@pg.gda.pl

Piotr Krzyślak, Assoc. Prof.

Faculty of Machines and Transportation

Poznan University of Technology

Piotrowo 3

60-965 Poznan, POLAND 